\title{
PHASE AND FREQUENCY LOCKED MAGNETRONS FOR SRF SOURCES*
}

\author{
M. Neubauer, R. P. Johnson, Muons, Inc. \\ M. Popovic, A. Moretti. FNAL, Batavia, IL U.S.A.
}

\begin{abstract}
Magnetrons are low-cost highly-efficient microwave sources, but they have several limitations, primarily centered about the phase and frequency stability of their output. When the stability requirements are low, such as for medical accelerators or kitchen ovens, magnetrons are the very efficient power source of choice. But for high energy accelerators, because of the need for frequency and phase stability---proton accelerators need 1-2 degrees source phase stability, and electron accelerators need .1-.2 degrees of phase stability---they have rarely been used. We describe a novel variable frequency cavity technique which will be utilized to phase and frequency lock magnetrons.
\end{abstract}

\section{INTRODUCTION}

In general, there are three different methods for stabilizing the magnetron frequency and phase. The first employs a method for coupling a more stable output circuit to the magnetron pi-mode resonator [1,2]. The second is by injecting a strong stable signal into the magnetron to force phase locking [5,6,7]. And the third is to create a feedback loop and adjust a controlling variable in the magnetron such as anode voltage or filament voltage or an active device in the output waveguide [4].

There are performance tradeoffs for all these techniques. In the case of a more stable resonator coupled to the magnetron, mechanical tuning is still required. For injection locking, large amounts of power are required: for example an $825 \mathrm{~W} 2.45 \mathrm{GHz}$ magnetron required $58 \mathrm{~W}$ and a $2 \mathrm{MW} 425 \mathrm{MHz}$ magnetron required $250 \mathrm{~kW}$ for injecton phase locking [5]. These large amounts of power make the injection magnetron effectively a low gain amplilfier of around $10 \mathrm{db}$. And efficiency is the tradeoff when changing the reactance of output waveguides.

Feedback schemes tend to have the greatest possibility, making the problem a low level RF design issue as long as the operating variable of the magnetron has a sufficient transfer function to affect tube performance [8]. To date those variables have been the anode and filament voltages.

\section{THE CONCEPT}

We propose a design where the variable is the pi-mode resonant structure locked into a feedback loop using ferrites in each cell. The permeability of the ferrite is adjusted by an external solenoid whose current is adjusted by a feedback circuit. The output of the magnetron is sampled by a coupler in the output waveguide, and compared to a local oscillator signal. The difference in phase and frequency is transformed into the current supplied to either all the solenoid-ferrite pairs or to individually selected solenoid-ferrite pairs to change their permeability. The change in permeability changes the frequency and phase of the pi-mode resonator to maintain stable operation and efficiency.

In addition, since the pi-mode structure frequency is variable, it is possible to construct a single magnetron that will work over a very broad frequency range.

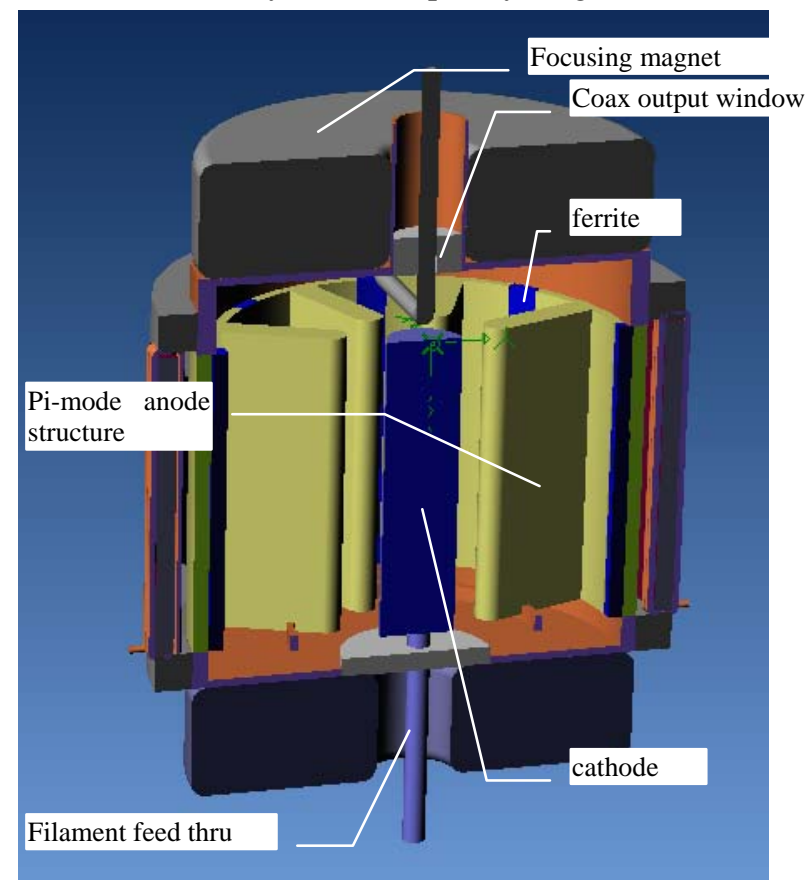

Figure 1: Cutaway of magnetron with ferrites in each cell of the pi-mode structure.

\footnotetext{
* Supported in part by USDOE Contract. DE-AC05-84-ER-40150 and by FRA DOE contract number DE-AC02-07CH11359 


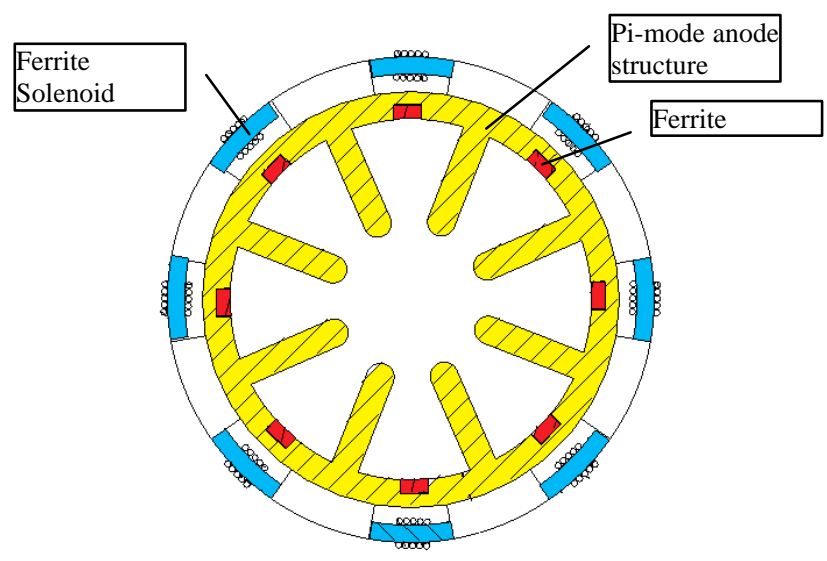

Figure 2: The pi-mode anode structure with ferrites, and solenoids

\section{VARIABLE FREQUENCY PI-MODE STRUCTURE}

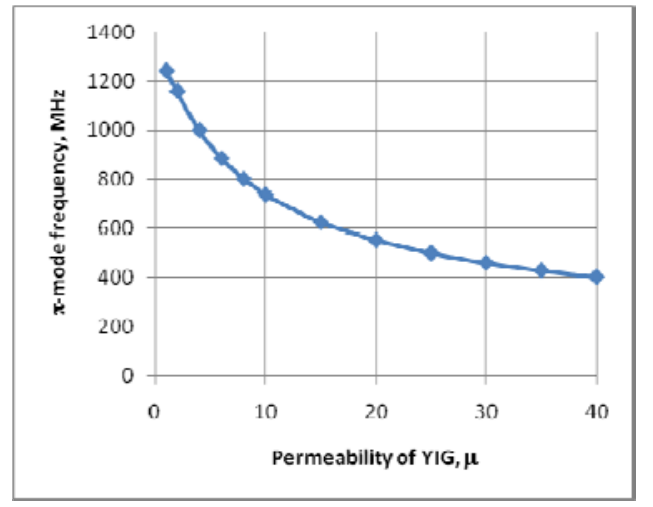

Figure 3: The pi-mode frequency as a function of the YIG ferrite permeability shown in Figure 6, for an 8-vane structure..

As shown in figure 3, the pi-mode frequency of an 8vane anode structure can be set to a wide range of frequencies by changing only the permeability of the ferrite material. The optimum design location for stabilization of the frequency is at the higher ranges of permeability where $\Delta \mu / \Delta \mathrm{f}$ is small. The 8-vane structure used in the Superfish calculations has five fundamental modes represented by $\pi / 8, \pi / 4, \pi / 2,3 \pi / 4$, and $\pi$ degrees of phase shift per cell. The pi-mode is shown in figure 4 for ferrites with a permeability of 8 . This is the typical operating mode for a magnetron.

The cross sectional dimension of the ferrite is $31 \times 22$ $\mathrm{mm}^{2}$. The outer radius of the anode is $136 \mathrm{~mm}$ and the inner radius $38 \mathrm{~mm}$. For $80 \%$ DC to RF efficiency, the Hartree voltage is $\mathrm{V}_{\mathrm{H}}=32 \mathrm{kV}$ with $\mathrm{B}=1500$ Gauss and cathode radius $12.7 \mathrm{~mm}$ [9].

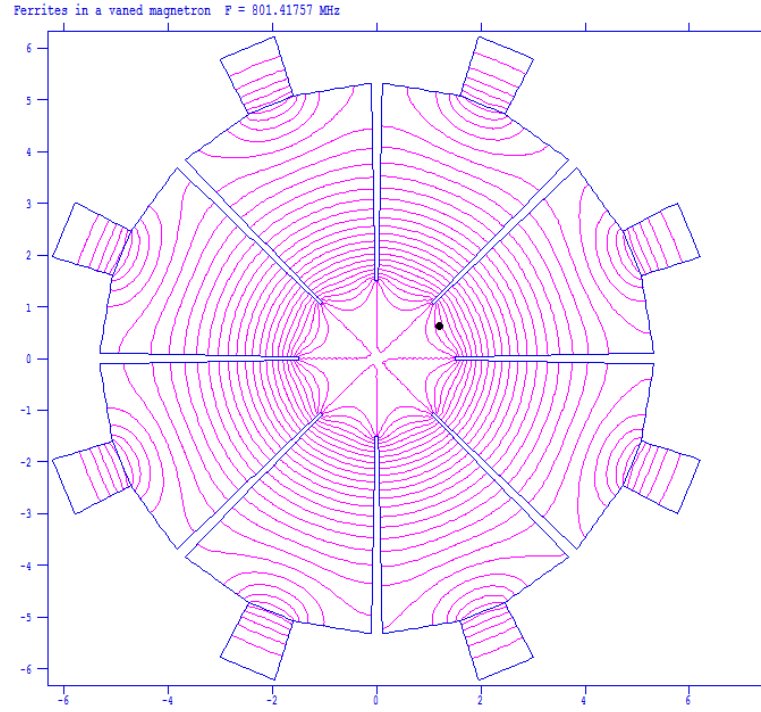

Figure 4: Superfish output showing an $801 \mathrm{MHz}$ pi-mode frequency in an anode structure with $\mu=8$.

For $\mu=40$, in the anode design of Figure 4, the operating frequency would be $400 \mathrm{MHz}$, and for the same magnetic field, $V_{H}=19 \mathrm{kV}$ for a DC to RF efficiency of $91 \%$.

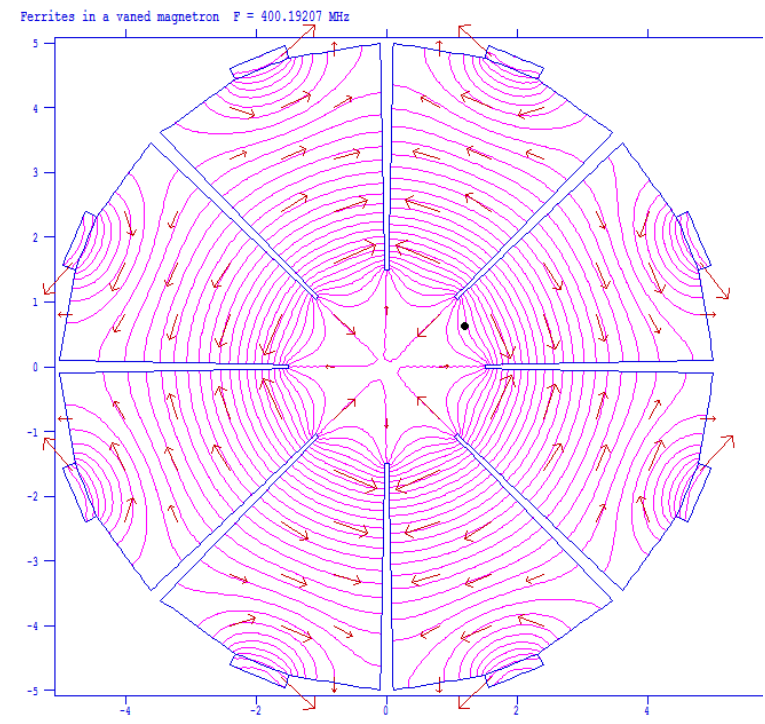

Figure 5: Superfish output showing $400 \mathrm{MHz}$ pi-mode using a ferrite such as NiZn with permeability 343 and a smaller cross section $5 \times 22 \mathrm{~mm}^{2}$.

One of the added advantages of individual solenoids controlling a ferrite in each cell, is the ability to change the separation between competing modes and tune out variations of the ferrite materials. In the $400 \mathrm{MHz}$ design, in Figure 5, a 6\% change in $\mu$ for two opposite cells doubles the separation between the $3 \pi / 4$, and $\pi$ mode from 4 to $8 \mathrm{MHz}$. 


\section{PERMEABILITY OF FERRITES}

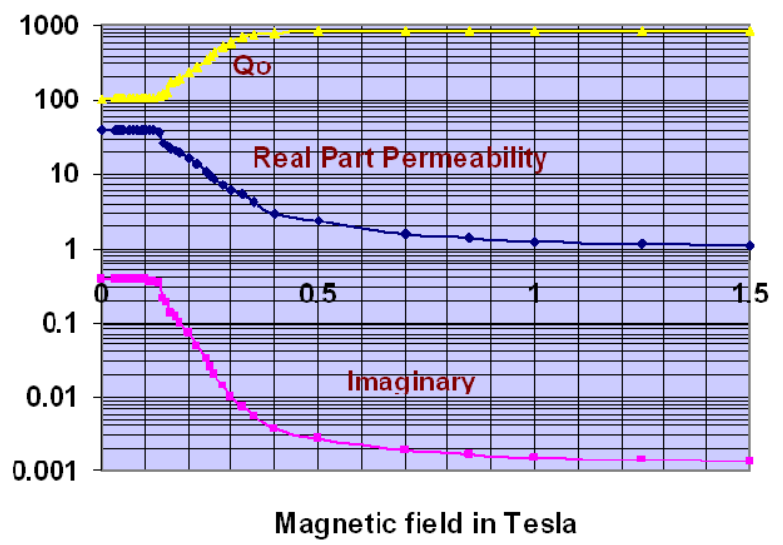

Figure 6: Measurements of permeability for a YIG material as a function of orthogonally biased magnetic field taken from reference [10].

Magnetic field orthogonal to the RF magnetic field has been shown to vary the permeability of materials slow enough for the tuning of the magnetron pi-mode structure in work down by Muons, Inc. in a prior Phase I program [10].

\section{FEEDBACK CIRCUIT}

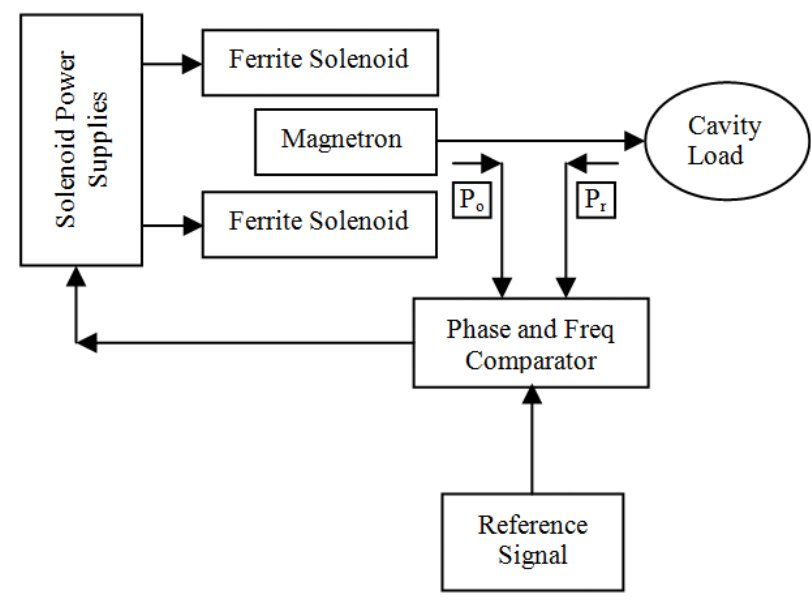

Figure 7: Block Diagram of fundamental modules of the feedback system for stabilizing the phase and frequency of the magnetron output.

The block diagram for a feedback circuit is shown in figure 7 . The output of the magnetron and the reflected signal from the load are fed to a phase and frequency comparator along with a reference signal. The algorithms in the comparator transform the differences between these signals into current controlled power supplies for the ferrites.

It is not clear how much phase control the reactance of the ferrites will produce. If $\Delta \mu$ is not sufficient then injection locking will be required. If the reference signal for injection locking is $30 \mathrm{db}$ down, then we have made significant improvement with a magnetron amplifier that has the gain of an IOT. If we can get to $60 \mathrm{db}$ down, then we have a magnetron amplifier with the gain of a klystron.

\section{CONCLUSIONS AND FURTHER STUDY}

The fundamental components of a system for stabilizing the phase and frequency of a magnetron have been presented, which include the frequency variable pimode anode circuit loaded with ferrites, the mechanism for changing the permeability of ferrites, and the feedback circuit required to control the system.

The optimum operating point for a ferrite loaded pimode structure needs to be further studied to find the optimum size and value of ferrite materials that will create small changes in frequency and phase.

The data presented show that a single ferrite loaded pimode structure could operate over a wide range of frequencies by simply adjusting the flux threading the ferrites. This innovation could be significant in applications from phased array radars to stabilized SRF sources.

What has not been studied is the effect of the lower Q of the pi-mode circuit. Since magnetrons operate in the large signal regime, lower Qs are not a significant problem as long as they are well matched in the coupling to the output RF coax. Heat generation from losses in the ferrites may be a problem, however, the need to remove the heat for frequency stabilization is no longer a requirement, since the feedback system will maintain the frequency of operation. The permeability of the ferrite is a strong function of temperature, and that also must be included in the feedback algorithm.

The interaction between the bias flux threading the ferrite and the electron trajectories must be considered. Recent advances in increasing the efficiency of magnetrons shows the advantage of pole pieces concentrating the focusing field close to the interaction region between the cathode and the vanes. This may be required to minimize effects of stray bias fields.

Competing modes in pi-mode structures and the frequency separation of those modes is critical to stable operation. In the studies already performed there seems to be ample flexibility in finding the optimum operating conditions as the effective radial length of the cells are changed relative to one another by changing the permeability of selected ferrites. The timing of the feedback circuit and the sources of phase and frequency excursion within the magnetron also need to be evaluated.

In conclusion, the use of ferrites threaded by controlled levels of magnetic flux orthogonal to RF magnetic fields inside of pi-mode structures offer new and significantly improved functionality for low $\mathrm{Q}$ devices such as a magnetrons.

\section{REFERENCES}

[1] J. O. Meilus, et. al., "Active Coaxial and Active Inverted Coaxial Magnetron Electronic Guns”; ISSN 1392-1215 Electronika, 2004. Nr.7(56) 
[2] Magnetrons with resonator element for stabilizing output radiation frequency, United States Patent 5017891

[3] Farney, G. K., "An Electronically-Tuned, Pulsed Coaxial Magnetron for Ku-Band”; Final rept. Jul 76Jul 77, on Phase 2; ADA063084

[4] Butler, J., Besse, S. B. "Development of a Prototype Ferrite Tuned Magnetron, QKH1707”, ADA000261, SEP 1974, RAYTHEON CO WALTHAM MASS MICROWAVE AND POWER TUBE DIV.

[5] Overett, T. et al., "Phase Locked Magnetrons as Accelerator RF Sources." Proceedings of the 12th IEEE Particle Accelerator Conference, 1987

[6] Chen, C., "Growth and Frequency Pushing Effects in Magnetron Phase-Locking,” PFC/JA-89-45, Plasma Fusion Center, MIT Cambridge, MA, October 1989

[7] Phongphaeth, P., Ph. D. dissertation, University of Michigan, 2007

[8] Tahir, I., "Phase and Frequency Locking of Magnetrons by Pushing and Pulling."

www.cockcroft.ac.uk/public/workshop-jul04/tahir.pdf

[9] Brown, W.C., "Satellite Power System (SPS) Magnetron Tube Assessment Study”, NASA Contract Report 3383, February 1981

[10] R. P. Johnson et al., this conference 